\title{
Hs.137007 is a novel epigenetic marker hypermethylated and up-regulated in breast cancer
}

\author{
TAE WOO KIM ${ }^{1}$, YOO JUNG KIM ${ }^{1}$, HYUN JI LEE ${ }^{1}$, SUN YOUNG MIN ${ }^{2}$, \\ HAN-SUNG KANG ${ }^{2}$ and SUN JUNG KIM ${ }^{1}$ \\ ${ }^{1}$ Department of Life Science, Dongguk Univ-Seoul, Seoul 100-715; ${ }^{2}$ Research Institute and Hospital, \\ National Cancer Center, Gyeonggi do 411-764, Korea
}

Received November 5, 2009; Accepted December 14, 2009

DOI: 10.3892/ijo_00000592

\begin{abstract}
This study was conducted to mine novel breastspecific unigenes and analyze their epigenetic regulation in breast cancer. Differential digital display and methylation analysis identified the Hs.137007 gene containing a Kelch domain as a candidate novel epigenetic marker. In 50 pairs of breast cancer tissues and nearby normal tissues the methylation level of the $14 \mathrm{CpG}$ sites at the promoter region $(-778$ to -485$)$ of the gene was higher in cancer tissues (72-93\%) than in normal tissues $(31-83 \%)$, with a high correlation rate $(\mathrm{p}<0.05)$. End-point RT-PCR and real-time RT-PCR revealed that Hs.137007 was up-regulated in cancer tissues. A clear relationship between high methylation levels and up-regulated expression was also observed in the cultured breast cell lines. The MCF7 (90-100\%) and MDAMB468 (100\%) cancer cell lines that showed higher methylation than the BT549 $(20-90 \%)$ and 184B5 (10-100\%) at the 14 CpGs also showed elevated gene expression. Taken together, these results indicate that the Hs.137007 gene is a novel gene specifically expressed in the breast that can be utilized as an epigenetic marker of breast cancer.
\end{abstract}

\section{Introduction}

Epigenetic change via alteration of the methylation status of the $\mathrm{CpG}$ sites has been known as an important regulation mecha-

Correspondence to: Dr Sun Jung Kim, Department of Life Science, Dongguk University, Seoul 100-715, Korea

E-mail: sunjungk@dongguk.edu

Dr Han-Sung Kang, Research Institute and Hospital, National Cancer Center, Gyeonggi do 411-764, Korea

E-mail: rorerr@ncc.re.kr

Abbreviations: $\mathrm{CpG}$, cytosine guanine dinucleotide; MSP, methylation specific PCR; RT-PCR, reverse transcription-polymerase chain reaction

Key words: digital differential display, unigene, Hs.137007, methylation, breast cancer nism of gene expression (1). Such changes are responsible for the development of cancer, tissue-specific gene expression, genomic imprinting and aging, and development (2-4). Indeed, many genes have been shown to be epigenetically regulated at the initiation or progression of breast cancer $(5,6)$. Two different categories of genes, proto-oncogene and tumor suppressor, are involved in epigenetic regulation leading to breast cancer. During the progression of cancer, proto-oncogenes such as Fen1, Igf2 and Pax2 (7-9) usually undergo hypomethylation at the $\mathrm{CpG}$ sites, which results in their upregulation. Conversely, tumor suppressors such as PCDH8, cystatin $\mathrm{M}$ and 15-hydroxyprostaglandin undergo hypermethylation that leads to the down-regulation of gene expression (10-12).

Methylation of the $\mathrm{CpG}$ sites prevents transcriptional activators from binding to their cis-acting elements, which inhibits target gene expression. Accordingly, induction of demethylation of the $\mathrm{CpG}$ sites in cancer cells such as MCF7 and MDAMB468 using the demethylating agent, 5-aza-2'deoxycytidine, recovered the gene expression to higher levels $(13,14)$. However, in sharp contrast to the majority of genes examined in this regard, some genes were found to be upregulated while the $\mathrm{CpG}$ sites were hypermethylated. For example, survivin was hypermethylated in endometrial cancer, and this was correlated with increased survivin expression (15). Additionally, methylation was found to inhibit the binding of p53, a repressor of survivin expression. In another case, it was found that the glycoprotein hormone $\alpha$-subunit, GPH $\alpha$, was hypermethylated in a variety of cell lines that produce proteins at high levels, while it was significantly less methylated in cell lines that produce the proteins at low levels (16).

Searching for genes that are epigenetically regulated in breast cancer has been conducted using several approaches. For example, individual tumor suppressors or proto-oncogenes that have $\mathrm{CpG}$ islands in their promoter were examined for changes in methylation in normal breast and cancer cells and tissues $(17,18)$. In addition, malignant cells cultured in the presence of 5-aza-2'-deoxycytidine and genes that showed altered expression before and after treatment were selected as candidates. Further analysis of the selected candidates revealed that CRIP1 and SAT were representative tumor suppressor genes (13). More recently, differential methylation chip analysis has been employed for the identification of differentially expressed genes in breast cancer. In this method, 
methylated DNAs are immunoprecipitated by anti-CpG antibody and then amplified by PCR (19). The use of this technique has led to the identification of differentially methylated genes such as TITF1 in endometrial cancer and WIT-1 in prostate cancer $(20,21)$.

This study was conducted to identify genes that are expressed in the breast in a tissue-specific manner and also regulated in an epigenetic manner. To accomplish this, we conducted digital differential display (DDD) (22), which led to the identification of Hs.137007. The gene was found to be hypermethylated in cancer tissues, while it was hypomethylated in normal tissues. Interestingly, expression of the gene was up-regulated in tissues as well as in cell lines that were hypermethylated. These results imply that the Hs.137007 gene could be used as a novel epigenetic marker in breast cancer.

\section{Materials and methods}

$D D D$. To identify candidates of the mammary gland-specific genes, digital differential display (http://www.ncbi.nlm.nih. gov/UniGene/info_ddd.shtml) was conducted. We compared expressed sequence tag (EST) libraries from human breast tissues and those from other various somatic tissues. Of the genes that were overrepresented in breast tissue-derived libraries, novel ESTs with structures and functions that have not yet been addressed were selected for further analysis.

The genomic sequence of Hs.137007 was identified using a human BLAST search (http://www.ncbi.nlm.nih.gov/ genome/seq/HsBlast.html). ESTs overlapping with Hs.137007 were located using the general BLAST program (http://www. ncbi.nlm.nih.gov/BLAST/). The open reading frame (ORF) and sequence alignments were identified using DNASIS.MAX version 2.0 for Windows (Miraibio, Alameda, CA). CpG islands were identified using the Sequence Manipulation Suite: CpG Islands (http://www.ualberta.ca/ stothard/ javascript/cpg_islands.html).

Cell culture and bisulfite treatment. The normal human breast cell line, 184B5, and three cancer cell lines, MCF7, MDAMB468 and BT549, were purchased from the American Type Culture Collection (ATCC; Manassas, VA) and grown in Dulbecco's modified Eagle's medium (DMEM) supplemented with $10 \%$ fetal bovine serum (FBS).

Genomic DNA extraction of tissue. Post-surgical samples of breast carcinoma were obtained, with written consent, from the National Cancer Center in Goyang, Korea. Nearby normal tissues were also collected for the control experiment. To isolate the chromosomal DNA, 50-100 mg of the tissue was extracted using a genomic DNA purification kit (Promega) according to the manufacturer's protocol. The extracted DNA was eluted with $250 \mu 1$ of distilled water.

Bisulfite genomic DNA sequencing. Bisulfite genomic DNA sequencing was conducted as previously described, with slight modification (23). Briefly, chromosomal DNA extracted from the tissues was subjected to bisulfite treatment using an EpiTect Bisulfite kit (Qiagen). The bisulfite-treated DNA was subjected to two rounds of polymerase chain reaction (PCR) to amplify the 346-bp promoter region (nucleotides
-778 to -485 ; transcriptional start site, +1$)$. The primer sequences used were 5'-GAAGGTTTTGGGGTTAAGGG-3' and 5'-CCTAAATAACCTCCAAAAAC-3' for the primary PCR, and 5'-GTTTTGGTTTAGTTTTAGAT-3' and 5'-CAA AACTTCAAATAAAAACC-3' for the nested PCR. In each case, the PCR conditions were $94^{\circ} \mathrm{C}$ for $2 \mathrm{~min}$, followed by 30 cycles of $94^{\circ} \mathrm{C}$ for $30 \mathrm{sec}, 55^{\circ} \mathrm{C}$ for $1 \mathrm{~min}$ and $72^{\circ} \mathrm{C}$ for $30 \mathrm{sec}$, with a final extension at $72^{\circ} \mathrm{C}$ for $5 \mathrm{~min}$. The resulting products were purified using a Qiaex II gel extraction kit (Qiagen) and then subjected to direct sequencing. The amplicons were also sub-cloned into the pGEM-T vector. The DNA sequences were confirmed by analyzing each PCR product in both directions. Primers 5'-AAACTTCAAATAA AAACCCC-3' and 5'-GTTTTGGTTTAGTTTTAGAT-3' were used for the direct sequencing. The investigated promoter region of Hs.137007 included $29 \mathrm{CpG}$ sites ( -778 to -485 GenBank accession no. NM 138433, taking the major transcriptional site to be +1 when numbering, Fig. 1A). The methylation ratio of each $\mathrm{CpG}$ site for each tissue was calculated as the percentage of methylation versus the methylated plus unmethylated sites.

Methylation-specific PCR. Primers specific to methylated (M)forward 5'-ATAGGGGTTTACGATGTCGG-3' and reverse 5'-TATAACCGACTACAACCGAC-3'-, or unmethylated (U)forward 5'-ATAGGGGTTTATGATGTTGG-3' and reverse 5'-TATAACCAACTACAACCAAC-3'- DNA from the Hs.137007 proximal promoter (118 bp; -691 to -573; transcriptional start site, +1 ) were used to amplify the samples in parallel with bisulfite-treated DNA samples from normal breast tissue and breast carcinomas. The PCR conditions for the methylation-specific PCR were 1 cycle at $94^{\circ} \mathrm{C}$ for $2 \mathrm{~min}$, followed by 20 cycles of $94^{\circ} \mathrm{C}$ for $30 \mathrm{sec}, 55^{\circ} \mathrm{C}$ for $1 \mathrm{~min}$ and $72^{\circ} \mathrm{C}$ for $30 \mathrm{sec}$, with a final extension at $72^{\circ} \mathrm{C}$ for $5 \mathrm{~min}$.

End-point RT-PCR. Total RNA from breast tissue or cell culture was prepared using Trizol according to the manufacturer's protocols (Gibco, BRL, Carlsbad, CA). Reverse transcription was conducted using $10 \mu \mathrm{g}$ of total RNA and a reverse transcription kit (Promega, Madison, WI). Expression was determined by PCR using primers deduced from the Hs.137007. The primers 5'-GGGCAACACCATTTACT GCC-3' and 5'-TTTTTTTTTTCAAAATAATC-3' were used for amplification of a 346-bp fragment. The following glyceraldehyde 3-phosphate dehydrogenase (GAPDH) primers were used to normalize expression: 5'-ACCACAGTCCATG CCATCAC and 5'-TCCACCACCCTGTTGCTGTA, which amplified a 452-bp cDNA fragment. End-point RT-PCR was conducted by subjecting the samples to 35 cycles of $94^{\circ} \mathrm{C}$ for $30 \mathrm{sec}, 55^{\circ} \mathrm{C}$ for $1 \mathrm{~min}$ and $72^{\circ} \mathrm{C}$ for $30 \mathrm{sec}$.

Real-time RT-PCR. Reverse transcription was performed as described above for the end-point RT-PCR. PCR reactions were conducted as described previously (24). Briefly, a master mix with a final volume of $20 \mu 1$ that was composed of $100 \mathrm{ng}$ of the template (reverse-transcribed cDNA), $300 \mathrm{nM}$ of each forward and reverse primer, a dye-labeled TaqMan probe and 1X PCR Master Mix (Applied Biosystems, Foster City, CA) was prepared. The samples were then subjected to an initial melt at $95^{\circ} \mathrm{C}$ for $10 \mathrm{~min}$, followed by 40 cycles 
A

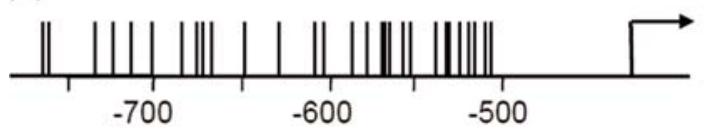

B
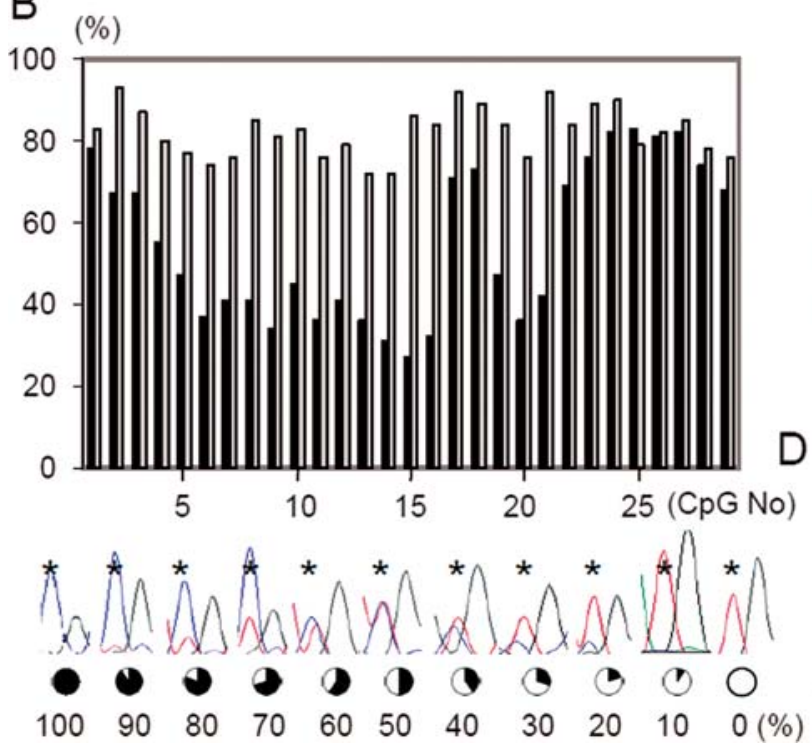

C CpG\#3
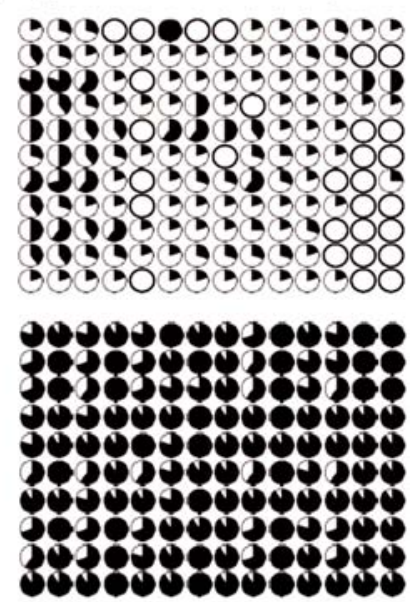

\section{8}
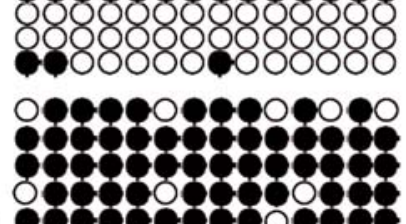

Figure 1. Methylation status of $\mathrm{CpG}$ islands at the promoter of $\mathrm{Hs} .137007$ in normal breast and cancer tissues. (A) Schematic diagram of the CpG islands at the promoter. The $29 \mathrm{CpG}$ sites examined are denoted by vertical lines. The arrow marks the transcription start site. (B) Methylation levels of the CpG islands in the normal breast (black bars) and cancer tissues (grey bars). (C) Methylation status determined by direct sequencing. Fourteen CpG sites from \#3 to 16 for 10 normal (top) and cancer (bottom) samples are presented. Sequencing diagrams corresponding to different methylation levels are presented below B. Asterisks indicate the peaks of overlapping $\mathrm{C}$ and T. (D) Methylation status determined by sequencing after subcloning of a PCR product into the pGEM-T plasmid. Top, normal; bottom, cancer.

of $95^{\circ} \mathrm{C}$ for $15 \mathrm{sec}$ and $60^{\circ} \mathrm{C}$ for $1 \mathrm{~min}$ in an ABI PRISM 7300 Sequence Detection System (Applied Biosystems). The partial cycle giving a statistically significant increase in the product was determined and normalized to GAPDH. The primers and probes used for Hs.137007 and GAPDH were synthesized by Applied Biosystems.

Statistical analysis. A $\chi^{2}$ test was used to analyze differences in the rate of each variable. A Student's t-test was used to detect differences in the mean values of the variables. P-values $<0.05$ were considered to be statistically significant. All calculations were performed using SPSS for Windows, release 7.0 (SPSS Inc.).

\section{Results}

In silico cloning and characterization of the human Hs.137007 gene. DDD was conducted to identify novel genes that were specifically expressed in the mammary gland with functions that had not yet been characterized. A total of 74,399 ESTs from 14 breast EST libraries were compared to 1,124,853 ESTs from 86 other tissues. As a result, dozens of novel ESTs that appeared almost exclusively in the breast unigene library were identified (Table I). Of the breast unigenes, ones that had $\mathrm{CpG}$ islands in the proximal promoter and showed a bias for the EST count toward either cancer or normal tissues due to a possible change in the methylation level were selected. For the three selected genes, Hs.137007, Hs.373787 and
Hs.613102, a frontier analysis of the promoter methylation level was conducted with 10 normal and cancer breast tissues. Among them, only Hs.137007 showed a remarkable change in methylation between the normal and cancer tissues, with a higher level being observed in the cancer tissues. Therefore, Hs.1370007 was finally chosen for extensive methylation analysis and identification of the relationship with gene expression.

The Hs.137007 EST identified was 3,008 bp long and located on human chromosome 22. A BLAST search revealed that Hs.137007 contained the Kelch domain, which was first found in the Drosophila Kelch protein (25) and later found in C. elegans and humans $(26,27)$.

Aberrant hypermethylation of the Hs.137007 gene in human breast tumors. A CpG island spanning from -778 to -485 and containing $29 \mathrm{CpG}$ sites was identified in the upstream region of the Hs.137007 gene (Fig. 1A). This 293-bp region was amplified by two rounds of nested PCR from bisulfitemodified DNA. The methylation status of the $\mathrm{CpG}$ sites was then determined by direct sequencing of the PCR product.

A total of 50 sets of breast cancer tissues and nearby normal tissues were collected for analysis of the promoter methylation and expression of Hs.137007. Pathological evaluation revealed that all cancer tissues were the ductal cancer type. Sequencing of the PCR products revealed that all cytosines were converted to thymines, except for those that existed as $\mathrm{CpG}$ doublets and were methylated. The methylation 
Table I. Top 10 unknown genes with highest enrichment in breast identified by digital differential display.

\begin{tabular}{|c|c|c|c|c|c|}
\hline \multirow[b]{2}{*}{ Unigene } & \multicolumn{2}{|c|}{ Frequency } & \multirow[b]{2}{*}{ Symbol } & \multirow[b]{2}{*}{$\begin{array}{l}\text { Enrichment } \\
\text { in cancer }\end{array}$} & \multirow[b]{2}{*}{$\begin{array}{l}\text { Presence of } \\
\text { CpG islands }\end{array}$} \\
\hline & Breast & Normal & & & \\
\hline Hs. 137007 & 0.00010 & 0 & Kelch domain containing 7B (KLHDC7B) & $\mathrm{C}$ & + \\
\hline Hs. 373787 & 0.00030 & 0 & Ankyrin repeat domain 30A (ANKRD30A) & $\mathrm{C}$ & + \\
\hline Hs. 350570 & 0.00030 & 0 & Dermcidin (DCD) & $\mathrm{C}$ & - \\
\hline Hs.129636 & 0.00020 & 0 & Transcribed locus & $\mathrm{N}$ & - \\
\hline Hs. 46452 & 0.00010 & 0 & Secretoglobin, family $2 \mathrm{~A}$, member 2 (SCGB2A2) & $\mathrm{C}$ & - \\
\hline Hs. 25345 & 0.00010 & 0 & Transcribed locus & $\mathrm{N}$ & - \\
\hline Hs.560938 & 0.00010 & 0 & Transcribed locus & $\mathrm{C}$ & - \\
\hline Hs. 586211 & 0.00010 & 0 & Transcribed locus & $\mathrm{C}$ & - \\
\hline Hs.652267 & 0.00010 & 0 & Transcribed locus & $\mathrm{C}$ & - \\
\hline Hs.611255 & 0.00010 & 0 & Transcribed locus & $\mathrm{C}$ & + \\
\hline
\end{tabular}

${ }^{\mathrm{a}} \mathrm{C}$, enriched in cancer tissue; $\mathrm{N}$, enriched in normal tissue. ${ }^{\mathrm{b}}$ Presence $(+)$ or absence (-) of $\mathrm{CpG}$ islands at the promoter region.

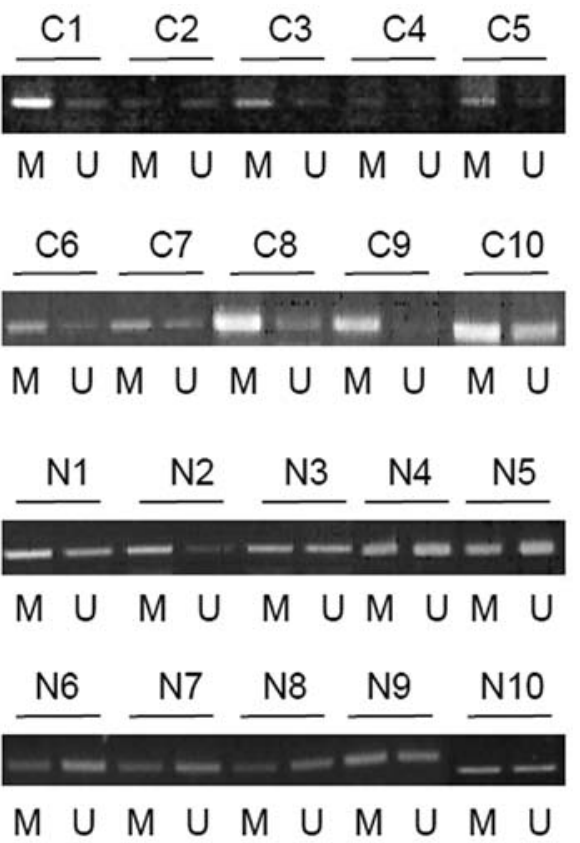

Figure 2. MSP analysis of the normal breast and cancer tissues. Results of MSP of 10 sets of cancer (C1-C10) and normal (N1-N10) tissues are shown. Lanes $\mathrm{M}$ and $\mathrm{U}$ represent amplification using primers specific for methylated cytosine and unmethylated cytosine, respectively.

level in the cancer tissues was higher or similar to that of normal breast tissues for all $\mathrm{CpG}$ sites. Fig. 1B shows the methylation level of each $\mathrm{CpG}$ site from CpG1 to CpG29 that showed higher methylation in the breast cancer tissue $(72-93 \%)$ than in the normal tissue $(31-83 \%)(\mathrm{p}<0.05)$. Notably, CpG6-CpG16 showed remarkable differences in methylation between the two groups: normal tissue, $32-46 \%$; cancer tissue, $68-83 \%$. The results of the direct sequencing were confirmed by sequencing of the PCR-amplified DNA after subcloning into the pGEM-T plasmid (Fig. 1C and D). Analysis of five sets of 10 plasmids for normal and cancer tissues indicated a similar methylation level in samples analyzed by direct sequencing and plasmid sequencing. Overall, the percentage of methylated $\mathrm{CpG}$ sites from 6 to 16 was $76 \%$ in the breast cancer tissues and $37 \%$ in the normal tissues, indicating that DNA from cancer exhibited higher average methylation levels.

To determine if cancer tissues could be discriminated from normal tissues by methylation-specific PCR (MSP) based on the methylation level, MSP was used to analyze the same sets of normal and cancer tissues. The results revealed that higher amounts of PCR product were amplified from the cancer tissues using the methylation-specific primers than the unmethylation-specific primers (Fig. 2). The results also indicated that samples that were found to have higher methylation levels by bisulfite-sequencing had a more remarkable difference in intensity between the cancer and normal breast tissues. However, no remarkable differences in the amount of PCR product produced using the methylationspecific primers and the unmethylation-specific primers were observed in the normal tissues (Fig. 2).

Increased Hs.137007 expression in breast cancer tissues is correlated with promoter methylation. To identify the correlation between the Hs.137007 promoter methylation and mRNA expression, we conducted end-point RT-PCR and quantitative real-time RT-PCR analysis for total RNAs isolated from 50 sets of cancer and normal breast tissues. End-point RT-PCR revealed that 25 cancer tissue samples and 7 normal tissue samples expressed the gene out of a total of 50 samples $(\mathrm{p}=0.042)$ (Fig. 3A and B). Additionally, real-time RT-PCR analysis confirmed that the gene was more highly expressed in cancer tissues than in normal tissues (Fig. 3C). Taken together, these findings indicate that the Hs.137007 gene was activated in cancer tissues in which epigenetic hypermethylation occurred in the promoter. 


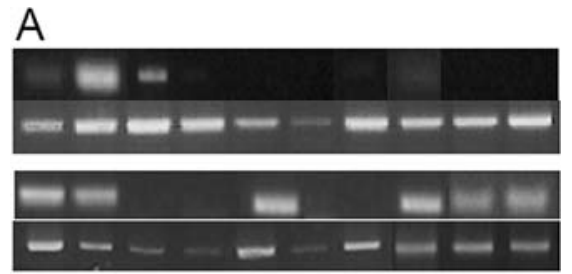

B

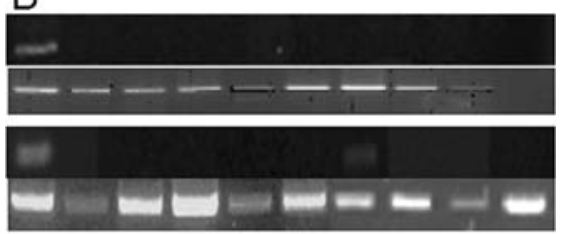

C

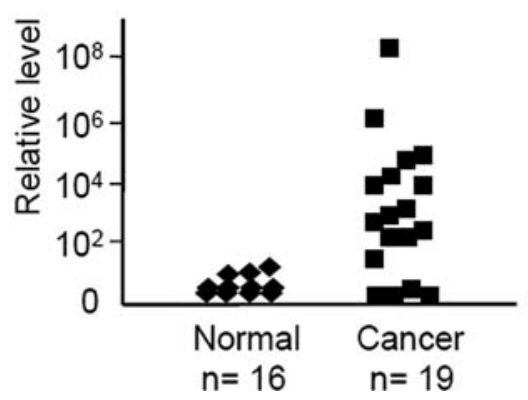

Figure 3. Expression analysis of Hs.137007 in cancer and normal breast tissues. End-point PCR for 20 sets of cancer (A) and normal (B) breast tissues The upper panel of each set is the 346-bp Hs.137007-specific RT-PCR product. The bottom panel is the 452-bp GAPDH product as a control. (C) Real-time RT-PCR. The relative expression levels are the averages of three independent reactions after normalization with GAPDH and are represented in log scale $(\mathrm{p}=0.042)$.

A

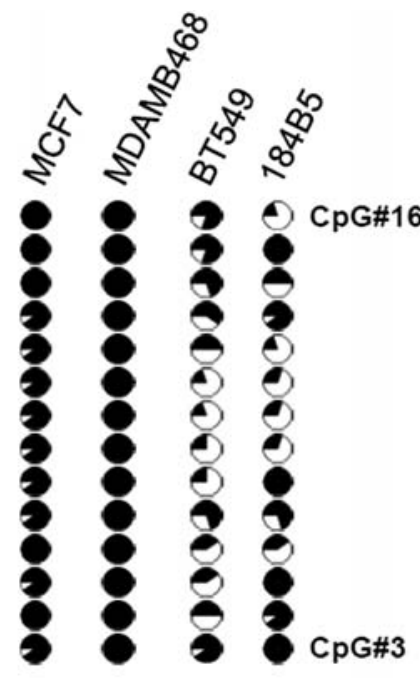

$\mathrm{B}$

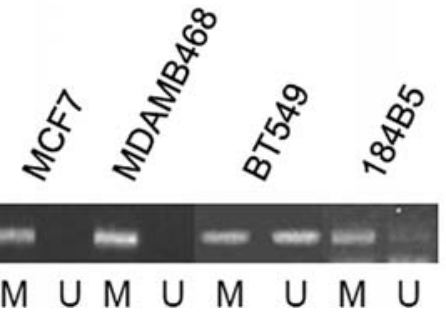

To further analyze the relationship between the promoter methylation and gene expression of Hs.137007, these characteristics were examined in a normal breast cell line and three cancer cell lines. In two cancer cell lines, MCF7 and MDAMB468, the $\mathrm{CpG}$ sites were highly methylated as in the cancer tissues; however, the $\mathrm{CpG}$ sites were less methylated in the BT549 cancer cell line and the 184B5 normal cell line (Fig. 4A and B). The expression of Hs.137007 in the two cell lines that showed higher methylation, MCF7 and MDAMB468, was higher than in the BT549 and 184B5 cell lines, which had a lower methylation level (Fig. 4C and D).

\section{Discussion}

In this study, we described the in silico identification of a novel breast-specific unigene, Hs.137007, and its epigenetic regulation in breast cancer. DDD analysis indicated that the gene expression was generally confined to the mammary gland, and that there was only minor expression in the blood, cervix, kidney and parathyroid. Additionally, no homologous genes were found by a BLAST search. One remarkable feature of the gene is that it contains a kelch domain. The kelch motif was first discovered as a 6-fold tandem element in the sequence of the Drosophila ORF1 protein. The repeated kelch motifs predict a conserved tertiary structure, a ß-propeller. Members of this growing superfamily, which are present throughout the cell and extracellularly, have diverse activities (28). However, the specific function of the protein as well as the kelch domain in the breast should be elucidated in further researches. We determined the methylation status of the $\mathrm{CpG}$ island by direct sequencing of the PCR product as well as sequencing of the

C

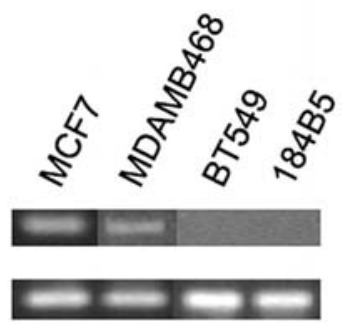

D

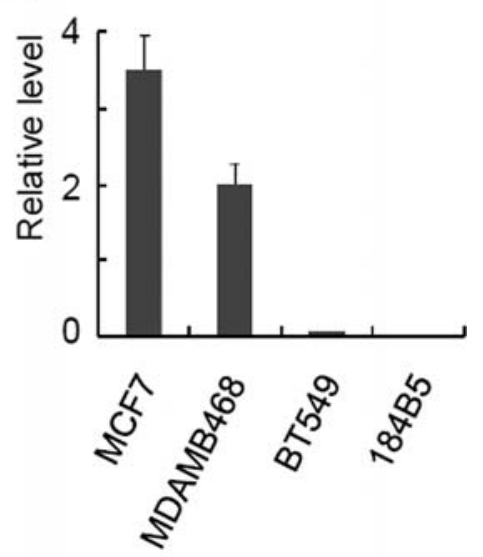

Figure 4. Relationship between methylation of the promoter and expression of Hs.137007 in breast cell lines. (A) Methylation status in three cancer cell lines, MCF7, MDAMB468 and BT549, and in the normal cell line, 184B5. (B) MSP analysis of the promoter region. M and U denote PCR reactions in which primers designed to detect methylated and unmethylated $\mathrm{CpG}$ were used, respectively. End-point (C) and real-time (D) RT-PCR analysis for cancer and normal cell lines. 
plasmid containing the PCR product. Both techniques were found to be useful and provided similar results. Recently, pyrosequencing, which is a technique that enables precise analysis of the methylation level, has gained popularity (29). However, this technique is not yet applicable to long sequences covering many $\mathrm{CpG}$ sites. In the present study, pyrosequencing of a few samples in a more restricted region revealed results similar to those obtained when direct sequencing was conducted (data not shown).

Of the $29 \mathrm{CpG}$ sites examined, sites from $\mathrm{CpG} \# 6$ to CpG\#16 showed remarkable hypermetylation in cancer tissue samples when compared to normal tissue samples, while the downstream $\mathrm{CpG}$ sites showed similar levels of methylation in cancer and normal tissues. Additionally, CpG\#6-16 were found to overlap with the plausible binding sites for transcription factors AP-2 and GCF. It is well known that AP-2 can repress its target genes in cooperation with co-repressors such as KCTD1 (30). Moreover, GCF (GC factor) binds to specific GC-rich sequences in the promoter and represses the transcription of genes including epidermal growth factor receptor and insulin-like growth factor-I receptor $(31,32)$. In this respect, it is speculated that hypermethylation in the breast prevents the repressor from binding to the $\mathrm{CpG}$ sites, thereby inducing up-regulated expression.

It should be noted that the cancer tissues showed higher levels of methylation at the promoter $\mathrm{CpG}$ island and higher levels of expression than the normal tissues. These data are supported by the results obtained when cultured cell lines where evaluated, which revealed that higher levels of methylation resulted in higher gene expression when compared to cell lines with lower methylation levels (Fig. 4). It is generally recognized that promoter methylation is related to gene silencing in breast cancer and other cancer types $(33,34)$. However, cases of higher methylation and higher expression patterns such as those observed in this study have been rarely reported to date. Binding of p53 that acts as a repressor on the survivin promoter is inhibited by methylation. Furthermore, it has been suggested that de-repression by methylation is a general mechanism of p53 regulation (15). In the case of glycoprotein hormone- $\alpha$ subunit $(\mathrm{GPH} \alpha)$, when $\mathrm{CpG}$ sites in the Alu repeat sequence were hypermethylated, the Alu-binding protein MeSABp50 could not bind to it and transcription was activated. However, no consensus sequences of Alu were observed in the Hs.137007 promoter region.

One of the remarkable characteristics of the Hs.137007 gene is that the methylation level in cancer tissue and nearby normal tissue is highly correlated $(\mathrm{p}<0.005)$. Indeed, of the 50 pairs of breast cancer tissues and normal tissues evaluated here, 45 showed higher methylation levels in the cancer tissues than in the normal tissues (Fig. 1). Therefore, the gene is expected to be useful as an epigenetic marker to diagnose breast cancer.

In conclusion, this study describes a unique approach of combined evaluation of novel tissue-specific unigene and epigenetic regulation in breast cancer. This method revealed that the Hs.137007 gene was hypermethylated and up-regulated in breast cancer tissues. Future studies should be conducted to annotate the function of the gene in the breast and to elucidate the molecular mechanism by which up-regulated expression in cancer occurs in response to hypermethylation.

\section{Acknowledgments}

The authors thank Dr K.H. Han and Dr W.I. Yang at Yonsei University for their kind support of the histological analysis of tissues.

\section{References}

1. Schones D and Zhao K: Genome-wide approaches to studying chromatin modifications. Nat Rev Genet 9: 179-191, 2008.

2. Fazzari $\mathrm{M}$ and Greally J: Epigenomics: beyond $\mathrm{CpG}$ islands. Nat Rev Genet 5: 446-455, 2004.

3. Bird A: DNA methylation patterns and epigenetic memory. Genes Dev 16: 6-21, 2002.

4. Blelloch R, Wang Z, Meissner A, Pollard S, Smith A and Jaenisch R: Reprogramming efficiency following somatic cell nuclear transfer is influenced by the differentiation and methylation state of the donor nucleus. Stem Cells 24: 2007-2013, 2006.

5. Widschwendter $\mathrm{M}$ and Jones P: DNA methylation and breast carcinogenesis. Oncogene 21: 5462-5482, 2002.

6. Rodenhiser D, Andrews J, Kennette W, et al: Epigenetic mapping and functional analysis in a breast cancer metastasis model using whole-genome promoter tiling microarrays. Breast Cancer Res 10: R62, 2008

7. Singh $\mathrm{P}$, Yang M, Dai H, et al: Overexpression and hypomethylation of flap endonuclease 1 gene in breast and other cancers. Mol Cancer Res 6: 1710-1717, 2008.

8. Ito Y, Koessler T, Ibrahim A, et al: Somatically acquired hypomethylation of IGF2 in breast and colorectal cancer. Hum Mol Genet 17: 2633-2643, 2008.

9. Wu H, Chen Y, Liang J, et al: Hypomethylation-linked activation of PAX2 mediates tamoxifen-stimulated endometrial carcinogenesis. Nature 438: 981-987, 2005

10. Yu J, Koujak S, Nagase S, et al: PCDH8, the human homolog of PAPC, is a candidate tumor suppressor of breast cancer. Oncogene 27: 4657-4665, 2008.

11. Ai L, Kim W, Kim T, et al: Epigenetic silencing of the tumor suppressor cystatin $\mathrm{M}$ occurs during breast cancer progression. Cancer Res 66: 7899-7909, 2006.

12. Wolf I, O'Kelly J, Rubinek T, et al: 15-hydroxyprostaglandin dehydrogenase is a tumor suppressor of human breast cancer. Cancer Res 66: 7818-7823, 2006.

13. Rivenbark A, Jones W, Risher J and Coleman W: DNA methylation-dependent epigenetic regulation of gene expression in MCF-7 breast cancer cells. Epigenetics 1: 32-44, 2006.

14. Miyamoto K, Fukutomi T, Akashi-Tanaka S, et al: Identification of 20 genes aberrantly methylated in human breast cancers. Int J Cancer 116: 407-414, 2005.

15. Nabilsi N, Broaddus R and Loose D: DNA methylation inhibits p53-mediated survivin repression. Oncogene 28: 2046-2050, 2009.

16. Cox G, Gutkin D, Haas M and Cosgrove D: Isolation of an Alu repetitive DNA binding protein and effect of $\mathrm{CpG}$ methylation on binding to its recognition sequence. Biochim Biophys Acta 1396: 67-87, 1998 .

17. Arapshian A, Kuppumbatti Y and Mira-y-Lopez R: Methylation of conserved $\mathrm{CpG}$ sites neighboring the beta retinoic acid response element may mediate retinoic acid receptor beta gene silencing in MCF-7 breast cancer cells. Oncogene 19: 4066-4070, 2000.

18. Witcher $M$ and Emerson B: Epigenetic silencing of the p16(INK4a) tumor suppressor is associated with loss of CTCF binding and a chromatin boundary. Mol Cell 34: 271-284, 2009.

19. Schumacher A, Kapranov P, Kaminsky Z, et al: Microarraybased DNA methylation profiling: technology and applications. Nucleic Acids Res 34: 528-542, 2006.

20. Zighelboim I, Goodfellow P, Schmidt A, et al: Differential methylation hybridization array of endometrial cancers reveals two novel cancer-specific methylation markers. Clin Cancer Res 13: 2882-2889, 2007.

21. Wang Y, Yu Q, Cho A, et al: Survey of differentially methylated promoters in prostate cancer cell lines. Neoplasia 7: 748-760, 2005.

22. Yang H, Cho E, Han K, Kim H and Kim S: Characterization of a novel mouse brain gene (mbu-1) identified by digital differential display. Gene 395: 144-150, 2007.

23. Kang J, Kim S, Noh D, Choe K, Lee E and Kang H: The timing and characterization of p53 mutations in progression from atypical ductal hyperplasia to invasive lesions in the breast cancer. J Mol Med 79: 648-655, 2001. 
24. Ji JW, Yang HL and Kim SJ: Analysis of cag-8: a novel poly $(\mathrm{Q})$-encoding gene in the mouse brain. Biochem Biophys Res Commun 346: 1254-1260, 2006.

25. Xue F and Cooley L: Kelch encodes a component of intercellular bridges in Drosophila egg chambers. Cell 72: 681693, 1993.

26. Ohmachi M, Sugimoto A, Iino Y and Yamamoto M: kel-1, a novel Kelch-related gene in Caenorhabditis elegans, is expressed in pharyngeal gland cells and is required for the feeding process. Genes Cells 4: 325-337, 1999.

27. Lai F, Orelli B, Till B, et al: Molecular characterization of KLHL3, a human homologue of the Drosophila kelch gene. Genomics 66: 65-75, 2000.

28. Adams J: Characterization of a Drosophila melanogaster orthologue of muskelin. Gene 297: 69-78, 2002.

29. Shaw R, Liloglou T, Rogers S, et al: Promoter methylation of P16, RARbeta, E-cadherin, cyclin A1 and cytoglobin in oral cancer: quantitative evaluation using pyrosequencing. Br J Cancer 94: 561-568, 2006.
30. Ding X, Luo C, Zhou J, et al: The interaction of KCTD1 with transcription factor AP-2alpha inhibits its transactivation. J Cell Biochem 106: 285-295, 2009.

31. Kitadai Y, Yamazaki H, Yasui W, et al: GC factor represses transcription of several growth factor/receptor genes and causes growth inhibition of human gastric carcinoma cell lines. Cell Growth Differ 4: 291-296, 1993.

32. Morrione A, De Angelis T and Baserga R: The GC factor regulates the expression of the insulin-like growth factor-I receptor. Cell Prolif 28: 659-671, 1995.

33. Huang T, Perry M and Laux D: Methylation profiling of $\mathrm{CpG}$ islands in human breast cancer cells. Hum Mol Genet 8: 459-470, 1999.

34. Costello J, Frühwald M, Smiraglia D, et al: Aberrant CpGisland methylation has non-random and tumour-type-specific patterns. Nat Genet 24: 132-138, 2000. 